\title{
Predicting Lamina Propria Invasion in Patients with Non- muscle-invasive Bladder Cancer: Do RDW and NLR Really Work?
}

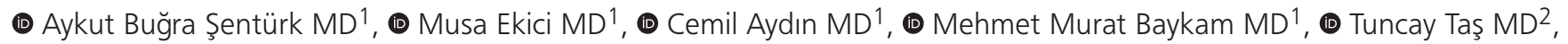 \\ (D) Ersan Arda $\mathrm{MD}^{3}$, (D) Basri Çakıroğlu MD4 \\ 1 Hitit University Faculty of Medicine, Department of Urology, Çorum, Turkey \\ 2 Esenyurt University Istanbul Surgical Hospital, Clinic of Urology, Istanbul, Turkey \\ 3 Trakya University Faculty of Medicine, Department of Urology, Edirne, Turkey \\ 4 Hisar Intercontinental Hospital, Clinic of Urology, Istanbul, Turkey
}

\begin{abstract}
Objective: To determine the role of preoperative neutrophil-to-lymphocyte ratio (NLR) and red cell distribution width (RDW) in predicting lamina propria invasion in bladder cancer.

Materials and Methods: Eighty-eight patients with non-muscle-invasive bladder cancer were evaluated retrospectively. The patients were divided into those with Ta tumors (group 1: $n=36$ ) and those with T1 tumors (group 2: $n=52$ ). For each patient, white blood cell, neutrophil, and leukocyte counts and RDW values were evaluated.

Results: NLR was significantly lower in patients with Ta tumors. In addition, NLR below 3.22 was associated with $80.6 \%$ probability of Ta disease. RDW sensitivity in Ta non-muscle-invasive bladder tumors was much higher compared to T1 tumors. RDW below 15.35 was associated with $94.4 \%$ probability of Ta disease.

Conclusion: NLR and RDW are basic blood parameters that physicians can assess easily. Our results indicate that a combination of NLR and RDW can help clinicians predict lamina propria invasion in non-muscle-invasive bladder tumors.

Keywords: Bladder cancer, superficial, lamina propria, invasion
\end{abstract}

\section{Introduction}

The incidence of bladder cancer has increased in recent years. Its prevalence increases with age and it is 3-4 times more common in men than in women. It is the sixth most common cancer in the United States of America and accounts for $8 \%$ of all cancers in men and $2 \%$ of all cancers in women (1). It is predicted that 76,960 new cases will be diagnosed and 16,390 deaths will be attributed to bladder cancer in 2016 (2). Seventyfive percent of newly diagnosed cases are limited to the mucosa ( $\mathrm{Ta}$, carcinoma in situ) or submucosa (T1), and these superficial bladder tumors have a high risk of recurrence and progression despite local treatment. The remaining $25 \%$ of newly diagnosed cases have muscle invasion and require a more radical approach such as surgery or radiotherapy (3).
Due to the high risk of recurrence and progression, risk scales have been developed to guide treatment planning. The European Organization for Research and Treatment of Cancer (EORTC) and Club Urológico Español de Tratamiento Oncológico (CUETO) risk models are most commonly used to predict a patient's risk of recurrence and progression. Although these tables are used frequently in clinical practice, they tend to overestimate the risks and have a low differentiation rate for prognostic results. As a result, new prognostic markers are needed to improve the predictive value of these risk evaluation tables (4). Previous studies have demonstrated the effect of systemic inflammation in the growth and progression of many types of cancer and shown that inflammation stimulates tumor angiogenesis, invasion, and metastasis (5). For example, in colorectal cancer patients who underwent potentially curative 
resection, systemic inflammatory response predicted poor outcome (6). A study by Kum et al. (7) showed that the inflammatory marker neutrophil-to-lymphocyte ratio (NLR) can be used to differentiate benign lesions from malign ones, especially for laryngeal lesions. Similar studies reported that NLR could be elevated in laryngeal cancer and may be a beneficial marker for diagnosis, detection of recurrence, and differentiation of malign and pre-malign lesions (8). Mano et al. (9) emphasized that elevated NLR was an independent predictor of progression and recurrence of non-muscle-invasive bladder cancers. In addition, NLR was shown to be associated with muscle invasion, extravesical disease, and worse diseasefree and overall survival $(10,11)$. Red cell distribution width (RDW) was also shown to be an independent prognostic factor in various cancers $(12,13)$. Aim of this study was to determine the role of preoperative NLR and RDW in predicting lamina propria invasion in bladder cancer.

\section{Materials and Methods}

This study was approved by a local ethics committee and conducted in accordance with the principles of the Declaration of Helsinki. The medical files of 88 patients diagnosed with non-muscle-invasive bladder cancer between January 2014 and January 2017 were evaluated retrospectively. Patients with proven preoperative infection, unexplained leukocytosis, or history of hematological malignancy, and those who were re-operated due to bladder cancer were excluded from the study. The patients included in the study were separated into two groups, those with Ta tumors (group 1: $n=36$ ) and T1 tumors (group 2: $n=52$ ). For each patient, white blood cell (WBC), neutrophil (N), and lymphocyte (L) counts and RDW values were evaluated and postoperative pathology reports were reviewed. Groups were compared according to their age, gender, WBC, N and L counts, NLR, and RDW values.

\section{Statistical Analysis}

Statistical analyses were performed using SPSS software package (version 22.0, SPSS Inc. Chicago, IL, USA; licensed to Hitit University). Distribution of normality was tested with the Kolmogorov-Smirnov test. Continuous variables were presented as mean \pm standard deviation or median (min-max) according to distribution, and categorical variables were expressed as numbers and percentages. Continuous variables were analyzed with Student's t-test to compare the means of normally distributed variables between two independent groups. Non normally distributed independent samples were compared using Mann-Whitney $U$ test. Logistic regression analysis was performed with an enter method to identify independent predictors of the Ta and $\mathrm{T} 1$ groups. Relationships between categorical variables were investigated by chi-square test. Receiver operating characteristic (ROC) curve analyses were conducted and areas under the ROC curves were calculated to evaluate the diagnostic accuracy of RDW and NLR in detecting lamina propria invasion. Sensitivity, specificity, positive predictive value (PPV), negative predictive value (NPV), and positive likelihood ratio were calculated for optimal cutoff values. A $p$ value $<0.05$ was used to determine the statistical significance.

\section{Results}

Patient characteristics (age, gender, tumor grade and size, origin, neutrophil count, lymphocyte count, platelet count, NLR, and RDW) are given Table 1.

After logistic regression analysis made using the Enter method; RDW and NLR were found to be statistically significant in differentiating $T 1$ from $T a(p=0.023$ and $p=0.050$ respectively; Table 2).

ROC analysis was performed to measure the classifying success of the logistic regression analysis. Area under curve was found to be $0.624(0.507-0.742)$ and $0.600(0.481-0.719)$ for RDW and NLR, respectively (Table 3 ). Cutoff points were determined as 15.35 for RDW and 3.22 for NLR by Youden index.

\begin{tabular}{|c|c|c|c|c|}
\hline & - & Group $1(\mathrm{Ta})$ & Group 2 (T1) & $\mathrm{p}$ value \\
\hline Age, years & - & $70.89 \pm 10.67$ & $69.67 \pm 12.33$ & $0.632^{\mathrm{b}}$ \\
\hline \multirow{2}{*}{ Gender } & Female (\%) & $1(11.1 \%)$ & $9(88.9 \%)$ & \multirow{2}{*}{$0.075^{\mathrm{a}}$} \\
\hline & Male (\%) & $35(44.9 \%)$ & $43(55.1 \%)$ & \\
\hline \multirow{2}{*}{ Grade } & High & $6(12.2 \%)$ & $43(87.8 \%)$ & \multirow{2}{*}{$<0.001^{\mathrm{a}}$} \\
\hline & Low & $30(78.9 \%)$ & $8(21.1 \%)$ & \\
\hline \multirow{2}{*}{ Tumor size } & $>3$ & $11(25.0 \%)$ & $33(75.0 \%)$ & \multirow{2}{*}{$0.002^{\mathrm{a}}$} \\
\hline & $<3$ & $25(58.1 \%)$ & $18(41.9 \%)$ & \\
\hline \multirow{2}{*}{ Origin } & Unifocal & $25(47.2 \%)$ & $28(52.8 \%)$ & \multirow{2}{*}{$0.171^{\mathrm{a}}$} \\
\hline & Multi-focal & $11(32.4 \%)$ & $23(67.6 \%)$ & \\
\hline Neutrophil count, median (minimum-maximum) & - & $5.21(2.66-12.60)$ & $4.69(1.80-15.21)$ & $0.466^{c}$ \\
\hline Lymphocyte count, mean \pm SD & - & $1.98 \pm 0.73$ & $1.95 \pm 0.71$ & $0.842^{\mathrm{b}}$ \\
\hline NLR, median (minimum-maximum) & - & $2.40(0.88-4.71)$ & $2.84(1.39-18.55)$ & $0.114^{c}$ \\
\hline Platelet count, median (minimum-maximum) & - & $241500(97000-509000)$ & $233000(30000-489000)$ & $0.561^{c}$ \\
\hline RDW, median (minimum-maximum) & - & $13.5(10.7-16.40)$ & $14.0(12.60-29.40)$ & $0.049^{c}$ \\
\hline
\end{tabular}




\begin{tabular}{|l|l|l|l|l|l|l|}
\hline \multicolumn{2}{|l}{$\begin{array}{l}\text { Table 2. Logistic regression analysis results for estimation of Ta-T1 } \\
\text { groups }\end{array}$} & B & SE & p value & OR & $\mathbf{9 5 \%}$ Cl for OR \\
\cline { 5 - 8 } & & & & & Lower & Upper \\
\hline RDW & 0.361 & 0.159 & $\mathbf{0 . 0 2 3 *}$ & $\mathbf{1 . 4 3 5}$ & 1.050 & 1.960 \\
\hline Lymphocyte & 1.034 & 0.695 & 0.137 & 2.812 & 0.721 & 10.971 \\
\hline Neutrophil & -0.510 & 0.273 & 0.062 & 0.600 & 0.351 & 1.025 \\
\hline NLR & 0.983 & 0.502 & $\mathbf{0 . 0 5 0 *}$ & $\mathbf{2 . 6 7 2}$ & 1.000 & 7.143 \\
\hline Platelets & 0.000 & 0.000 & 0.782 & 1.000 & 1.000 & 1.000 \\
\hline $\begin{array}{l}\text { OR: Odds ratio, SE: Standard error of beta coefficient, Cl: Confidence interval, } \\
\text { RDW: Red cell distribution width, NLR: Neutrophil to lymphocyte ratio }\end{array}$ \\
\hline
\end{tabular}

Table 3. Receiver operating characteristic curve results

\begin{tabular}{|l|l|l|}
\hline & RDW & NLR \\
\hline AUC $(95 \% \mathrm{Cl})$ & $0.624(0.507-0.742)$ & $0.600(0.481-0.719)$ \\
\hline Cut-off & $\leq 15.35$ & $\leq 3.22$ \\
\hline Sensitivity & $0.294(0.179-0.440)$ & $0.451(0.313-0.595)$ \\
\hline Specificity & $0.944(0.800-0.990)$ & $0.806(0.634-0.912)$ \\
\hline PPV & $0.882(0.622-0.979)$ & $0.767(0.572-0.894)$ \\
\hline NPV & $0.485(0.365-0.607)$ & $0.509(0.374-0.642)$ \\
\hline LR+ & $5.29(1.28-21.73)$ & $2.32(1.12-4.82)$ \\
\hline AUC: Area & &
\end{tabular}

AUC: Area under curve, PPV: Positive predictive value, NPV: Negative predictive value, LR+: Positive likelihood ratio, $\mathrm{Cl}$ : Confidence interval, RDW: Red cell distribution width, NLR: Neutrophil to lymphocyte ratio

\section{Discussion}

Non-muscle-invasive bladder cancer accounts for $70 \%$ of all bladder cancers. Recurrence and progression of superficial bladder tumors, which is a very heterogeneous group, is basically related to tumor grade, number of tumor foci, and tumor size. EORTC risk classification is a tool to predict prognosis of superficial bladder cancer, but recent studies have shown that new risk predictors are needed to improve this prognostic risk classification $(14,15)$.

NLR and RDW are practical, easy to use, and accessible laboratory methods. In our study, we evaluated the role of NLR and RDW in predicting lamina propria invasion in patients with superficial bladder tumor who have undergone TUR.

Inflammation associated with cancer has been evaluated in many studies. Essentially, inflammation and cancer are linked by intrinsic and extrinsic mechanisms (5). Intrinsic mechanisms are related to tumor suppressor gene deactivation and oncogene activation, whereas the extrinsic mechanisms are activated via chronic inflammation or infection (16). Some prognostic scoring systems based on inflammation have been developed in order to predict prognosis. Glasgow prognostic scoring, based on C-reactive protein (CRP) and albumin levels, is the most widely known (16). Similarly, pre-treatment NLR is associated with systemic inflammation, and has been shown to have prognostic value and correlate with poor survival outcomes in various cancers.

In malignant diseases, the increase in WBC count is mostly due to cancer-associated myeloproliferation. Cancer cells stimulate myelopoesis and also impair myeloid cell differentiation, thus leading to elevated WBC count. The resulting increase in immature myeloid cells causes immunosuppression of myeloid suppressor cells and consequently triggers tumor progression and metastasis (16).

The relation between cancer and neutrophilia/lymphocytopenia has been demonstrated in various studies (17). Cytotoxic immunity is considered a natural defense mechanism against tumors, and malfunctions in this mechanism can result in disease stage progression and poor prognosis. In patients with advanced cancer, tumor prognosis is strongly correlated with leukocytosis, lymphocytopenia, and CRP (18). In addition, pretreatment NLR was shown to be associated with prognosis in many cancers. Especially in superficial bladder cancer, there are quite a few studies demonstrating the relation between NLR, recurrence, and progression. $(9,11,19)$

Consistent with the literature, our results showed that NLR was significantly lower in Ta disease. Furthermore, at NLR lower than 3.22 , the probability of disease being stage Ta was found to be $80.6 \%$.

Parameters related to inflammation such as WBC count, NLR, and RDW have been associated with prognosis in many cancers, but to the best of our knowledge, there have been no studies evaluating the combination of these markers in bladder cancer. RDW is a standard parameter measuring the variability in the size of erythrocytes. It is increased in cardiac diseases in particular but also in systemic inflammation. Rise in RDW can be associated with many types of cancer. In a study by Riedl et al. (12), increase in RDW was associated with poorer overall survival in breast, lung, colon, pancreas, prostate, brain, kidney, and stomach cancers. Likewise, in a study specifically investigating RDW values in colon cancer and colonic polyps, RDW was determined to be significantly higher in colon cancer patients (20).

Although the mechanism is not completely understood, it is believed that RDW is associated with IL-6, tumor necrosis factor, hepcidin, and other cytokines in the circulation which affect the biological behavior of tumor cells $(21,22)$. Moreover, RDW was shown to be associated with poor nutritional status and indicates the nutritional status of patients along with iron, folate, and vitamin B12 (23). A relationship between RDW and IGF-1, the cornerstone of metabolic aging and longevity, has also been reported (24).

In our study, the success rate for determining the Ta group was higher than that for the T1 group. RDW was found to be significantly lower in low-grade superficial bladder tumors. In other words, RDW sensitivity was much higher in Ta superficial bladder tumors compared to T1 tumors. In addition, RDW lower than 15.35 was associated with a $94.4 \%$ probability of stage Ta disease. Although the prognostic value of RDW has been demonstrated previously in various cancers, to the best of our knowledge this is the first study to evaluate the utility of RDW in predicting progression in patients with superficial bladder cancer.

\section{Study Limitations}

Limitations of the study include being single-center and retrospective and having a small sample size. Larger scale, prospective, multicenter studies will yield more reliable results. 


\section{Conclusion}

NLR and RDW are basic blood parameters that physicians can easily assess. This study suggests that the combination of NLR and RDW can help clinicians predict lamina propria invasion in superficial bladder tumors.

\section{Ethics}

Ethics Committee Approval: Retrospective study.

Informed Consent: Retrospective study.

Peer-review: Externally peer-reviewed.

\section{Authorship Contributions}

Concept: A.B.Ş., C.A., M.M.B., Design: A.B.Ş., M.E., Data Collection or Processing: A.B.Ş., Analysis or Interpretation: A.B.Ş., E.A., Literature Search: A.B.Ş., T.T., Writing: A.B.Ş.

Conflict of Interest: No conflict of interest was declared by the authors.

Financial Disclosure: The authors declared that this study received no financial support.

\section{References}

1. Tiu A, Jenkins LC, Soloway MS. Active surveillance for low-risk bladder cancer. Urol Oncol 2014;32:33.

2. Siegel RL, Miller KD, Jemal A. Cancer statistics, 2016. CA Cancer J Clin 2016;66:7-30.

3. Burger M, Catto JW, Dalbagni G, et al. Epidemiology and risk factors of urothelial bladder cancer. Eur Urol 2013;63:234-241.

4. Isharwal S, Konety B. Non-muscle invasive bladder cancer risk stratification. Indian J Urol 2015;31:289-296.

5. Mantovani A, Allavena P, Sica A, Balkwill F. Cancer-related inflamation. Nature 2008;454:436-444.

6. McMillan DC, Canna K, McArdle CS. Systemic inflammatory response predicts survival following curative resection of colorectal cancer. $\mathrm{Br}$ ] Surg 2003;90:215-219.

7. Kum RO, Ozcan M, Baklaci $D$, et al. Elevated neutrophil-tolymphocyte ratio in squamous cell carcinoma of larynx compared to benign and precancerous laryngeal lesions. Asian Pac J Cancer Prev 2014;15:7351-7355.

8. Duzlu M, Karamert R, Tutar $H$, et al. Neutrophil-lymphocyte ratio findings and larynx carcinoma: a preliminary study in Turkey. Asian Pac J Cancer Prev 2015;16:351-354.

9. Mano R, Baniel J, Shoshany O, et al. Neutrophil-to-lymphocyte ratio predicts progression and recurrence of non-muscle-invasive bladder cancer. Urol Oncol 2015;33:67.

10. Ceylan C, Doluoglu OG, Keleş I, et al. Importance of the neutrophilto-lymphocyte ratio in muscle-invasive and non-muscle invasive bladder tumors. Urologia 2014;81:120-124.
11. Krane LS, Richards KA, Kader AK, et al. Preoperative neutrophil/ lymphocyte ratio predicts overall survival and extravesical disease in patients undergoing radical cystectomy. J Endourol 2013;27:1046-1050.

12. Riedl J, Posch F, Königsbrügge $\mathrm{O}$, et al. Red cell distribution width and other redblood cell parameters in patients with cancer: association with risk of venous thromboembolism and mortality. PLoS One 2014;9:e111440.

13. Yilmaz A, Malya F, Ozturk G, et al. Effect of pre-operative red blood cell distribution on cancer stage and morbidity rate in patients with pancreatic cancer. Int J Clin Exp Med 2014;7:3072-3075.

14. Busato Júnior WF, Almeida GL, Ribas CA, et al. EORTC Risk Model to Predict Progression in Patients With Non-Muscle-Invasive Bladder Cancer: Is It Safe to Use in Clinical Practice? Clin Genitourin Cancer 2016;14:176-182.

15. Cimen HI, Halis F, Saglam HS, Gokce A. Can neutrophil to lymphocyte ratio predict lamina propria invasion in patients with non muscle invasive bladder cancer? Int Braz J Urol 2017;43:67-72.

16. Cheng YC, Huang CN, Wu W], et al. The Prognostic Significance of Inflammation Associated Blood Cell Markers in Patients with Upper Tract Urothelial Carcinoma. Ann Surg Oncol 2016;23:343-351.

17. Paramanathan A, Saxena A, Morris DL. A systematic review and meta-analysis on the impact of pre-operative neutrophil lymphocyte ratio on long term outcomes after curative intent resection of solid tumours. Surg Oncol 2014;23:31-39.

18. Maltoni M, Caraceni A, Brunelli Cet al. Prognostic factors in advanced cancer patients: evidence-based clinical recommendations--a study by the Steering Committee of the European Association for Palliative Care. J Clin Oncol 2005;23:6240-6248.

19. Ozyalvacli ME, Ozyalvacli G, Kocaaslan R, et al. Neutrophillymphocyte ratio as a predictor of recurrence and progression in patients with high-grade pT1 bladder cancer. Can Urol Assoc J 2015;9:126-131.

20. Ay S, Eryilmaz MA, Aksoy N, et al. Is early detection of colon cancer possible with red blood cell distribution width? Asian Pac J Cancer Prev 2015; 16:753-756.

21. Rhodes $C$ ), Howard LS, Busbridge $M$, et al. Iron deficiency and raised hepcidin in idiopathic pulmonary arterial hypertension: clinical prevalence, outcomes, and mechanistic insights. J Am Coll Cardiol 2011;58:300-309.

22. de Gonzalo-Calvo D, de Luxán-Delgado B, Rodríguez-González $S$, et al. Interleukin 6 , soluble tumor necrosis factor receptor I and red blood cell distribution width as biological markers of functional dependence in an elderly population: a translational approach. Cytokine 2012;58:193-198.

23. Tetè $S$, Nicoletti $M$, Saggini $A$, et al. Nutrition and cancer prevention. Int J Immunopathol Pharmacol 2012;25:573-581.

24. Podhorecka M, Halicka D, Szymczyk A, et al. Assessment of red blood cell distribution width as a prognostic marker in chronic lymphocytic leukemia. Oncotarget 2016;7:32846-32853. 